\title{
On the Use of Reciprocal Filter against WiFi packets for passive radar
}

\author{
Fabiola Colone, Francesca Filippini, Marco Di Seglio, Kevin Chetty
}

\begin{abstract}
This paper aims at a critical review of the signal processing scheme used in WiFi-based passive radar in order to limit its complexity and enhance its suitability for short range civilian applications. To this purpose the exploitation of a reciprocal filtering strategy is investigated as an alternative to conventional matched filtering at the range compression stage. Along with the well-known advantage of a remarkable sidelobes control capability for the resulting range-Doppler response, the use of a reciprocal filter is shown to provide additional benefits for the specific sensor subject of this study. Specifically, it allows to streamline the disturbance cancellation stage and to implement a unified signal processing architecture which is capable to handle the different modulation schemes typically adopted in WiFi transmissions. Appropriate adjustments are also proposed to the theoretical reciprocal filter in order to cope with the inherent loss in term of signal-to-noise power ratio. The effectiveness of the revised signal processing scheme encompassing the reciprocal filtering strategy is proved against both simulated and experimental datasets.
\end{abstract}

Keywords-passive radar, WiFi signals, short range surveillance, range compression, multipath removal.

\section{INTRODUCTION}

The concept of passive radar (PR) has been mostly investigated for long-range defense applications by parasitically exploiting the high-power transmitters of radio and television broadcast [1]-[3]. However, aiming at monitoring critical infrastructures and/or small private or public areas, several studies have also looked at the use of alternative RF emitters able to guarantee the persistent illumination of the areas of interest, though with a limited power density [4][5]. Such emitters include satellite-based transmitters for communication, broadcast and navigation services, which potentially provide global coverage [6]-[9]. However, a quite interesting alternative is offered by the transmitters for networking, which are proliferating at rapid rate and may allow the implementation of a PR for the surveillance of metropolitan and local areas, there including indoor applications [10]-[15]. Noteworthy, the exploitation of such illuminators of opportunity paves the way to the emerging technology of integrated sensing and communication (ISAC) systems, critical to addressing increased congestion of the RF spectrum [16].

F. Colone, F. Filippini and M. Di Seglio are with the Department of Information Engineering, Electronics and Telecommunications (DIET), Sapienza University of Rome, 00184 Rome, Italy.

email: \{fabiola.colone, francesca.filippini, marco.diseglio\}@uniroma1.it

$\mathrm{K}$. Chetty is with Department of Security \& Crime Science. University College London, Torrington Place, London, UK.

email: k.chetty@ucl.ac.uk
With particular reference to local area and indoor applications, the use of modern WLAN transmissions represents the most effective solution. In [17]-[25] and related works, the suitability of WiFi signals as waveforms of opportunity for PR has been investigated and appropriate signal processing strategies have been proposed for detecting and localizing moving targets against the competing background, namely the direct signal from the transmitter, its multipath replicas and the receiver noise. The effectiveness of such approaches has been proved in several experimental tests against drones, vehicles and people.

However, much still needs to be done to enable practical implementation of WiFi-based PR in civilian applications where low-cost, compactness and easy deployment represent the driving factors in the system design. To this purpose, commercial-off-the-shelf (COTS) components should be preferably employed together with appropriate architectures and signal processing strategies able to limit the system complexity. In fact, given the large bandwidth of the waveforms of opportunity and the variety of IEEE 802.11 based Standards that can be adopted at the physical layer level, quite demanding requirements could be set in terms of computational complexity when operating with conventional approaches.

In this paper, we take this perspective and we investigate possible modifications to the conventional signal processing scheme presented in [18] aimed at reducing the computational cost and the complexity of the signal processing architecture. Special attention is devoted to the most demanding processing blocks dedicated to the adaptive cancellation of unwanted signal contributions from the transmitter and the stationary scene as well as the rangeDoppler map evaluation, especially the range compression stage. Since these processing stages are modulation dependent, they require multiple, parallel, processing chains to be implemented, tailored to the Standard adopted for the transmitted packets [18],[26]-[29].

Therefore, we first reorganize the corresponding signal processing blocks more efficiently based on the characteristics of the WiFi transmissions, which are of a pulsed type, in order to reduce the computational load and simplify the data management.

Then we resort to a reciprocal filter $(\mathrm{RpF})$ in lieu of a conventional matched filter (MF) to implement the range compression stage. Such approach has been investigated in PR applications as a mean to mitigate the undesired sidelobes appearing in the signal Ambiguity Function (AF) when digital transmissions are used, which are caused by periodic 
and/or deterministic components in the adopted modulation schemes, especially with OFDM transmissions (e.g. pilot tones and guard intervals) [30]-[34]. Also, its benefits have been demonstrated for OFDM-based PR on moving platforms for ground moving target indication (GMTI) due to its capability to reduce the effects of the data-dependent variability of the system impulse response on the clutter cancellation stage [35].

In this paper, the use of $\mathrm{RpF}$ is investigated for WiFi-based PR exploiting different modulation schemes, there including OFDM and DSSS [36]. To this purpose, its application is not constrained to the framing of the emitted packets but rather performed on a packet-by-packet basis without any prior assumption and/or knowledge of the adopted Standard. This approach enables the design of a unified processing architecture which does not dependent on the specific modulation adopted at each packet and is capable to handle mixed transmissions, which are typical of WLANs connecting users with different requirements. Moreover, the use of the $\mathrm{RpF}$ is shown to simplify the adaptive estimation of the cancellation filter coefficients [37] thus reducing the corresponding computational cost. Appropriate supervised approaches are also proposed for an effective implementation of the RpF in order to cope with the inherent signal-to-noise power ratio (SNR) loss with respect to the MF. The effectiveness of the resulting WiFi-based PR signal processing scheme is proved against simulated data and then verified with experimental tests.

The paper is organized as follows. The conventional processing scheme for a WiFi-based PR is recalled in Section II where also a revised signal processing architecture is illustrated, in which the most demanding signal processing blocks are rearranged more effectively. The range compression based on a $\mathrm{RpF}$ is introduced in Section III and its benefits on the overall processing scheme are investigated, above all the sidelobe control and the clutter cancellation stages. In Section IV we propose appropriate supervised strategies for $\mathrm{RpF}$ implementation needed to control the expected SNR loss and we extensively investigate their robustness. Section V reports the results obtained with the derived processing scheme against experimental data. Finally, we draw our conclusions in Section VI.

\section{CRITICAL REVIEW OF THE CONVENTIONAL WIFI- BASED PR PROCESSING SCHEME}

A WiFi-based passive radar sensor exploits the WiFi access point (AP) as an illuminator of opportunity and detects moving objects by properly processing the signal $s(t)$ collected by the surveillance antenna. Since the exploited signal is of a pulsed type, namely it takes non-zero values only at given time periods corresponding to the transmission of Wi-Fi packets, we arrange in a sequence $s^{(p)}[n]$ the samples of the surveillance signals corresponding to the $p$-th packet transmission, i.e.

$$
\begin{gathered}
s^{(p)}[n]=s\left(N_{0}(p) / f_{s}+n / f_{s}\right) \\
n=0, \ldots, N_{s}(p)-1, p=0, \ldots, N_{P}-1
\end{gathered}
$$

where

- $\quad f_{s}$ is the sampling frequency;

- $\quad N_{0}(p)$ is the number of samples elapsed from the start of the observation up to the reception of the first sample of the $p$-th packet;

- $\quad N_{s}(p)$ is the number of samples within the $p$-th packet and we assume that different packets may have different durations;

- $N_{p}$ is the number of packets contained in the considered coherent processing interval (CPI).

Despite the WiFi signal is of a pulsed type, the short-range operation implies the direct signal from the AP, its multipath replicas, and the Doppler shifted echoes from potential targets to be simultaneously received by the sensor. Consequently, appropriate signal processing techniques are required to extract the weak target echo from the competing background and to estimate its position [18][20][37]. Moreover, the required signal processing techniques might be modulation dependent [26]-[28].

A complete signal processing chain is sketched in Fig. 1 for a passive radar exploiting WiFi packets with mixed modulation schemes as typically emitted by an 802.11 AP implementing different Standards. It can be conceptually divided into three main stages as illustrated in the following.

\section{1) Pre-processing stage}

First of all, the WiFi packets are identified and extracted from the received data stream. Then the adopted modulation scheme is recognized which allows different packets to be routed to the most appropriate processing chain.

In order to guarantee an effective operation for the PR, a good copy of the signal emitted by the AP should be made available at the receiver, namely the reference signal. In the considered short range application, there are two different ways to obtain a clean reference waveform ([15][18]).

i. If the transmitter of opportunity is directly accessible, a solution is to spill the signal from the AP itself, e.g., by means of a directional coupler inserted between the AP and its antenna. This approach is well suited for monostatic (or quasi-monostatic) configurations but, whilst providing quite a good copy of the signal of opportunity, it requires an additional receiving channel to be used to collect the reference signal.

ii. If the WiFi router in not accessible, then the reference waveform must be extracted from the signal collected by the surveillance antenna. Specifically, the transmitted signal can be reconstructed by demodulating and re-modulating the received signal packet $s^{(p)}[n]$ according to the employed IEEE 802.11 Standard. This approach can be implemented also in bistatic configurations and it avoids the need for a dedicated reference channel; however, it may suffer from reconstruction errors, especially in unfavourable multipath conditions.

For illustration purposes, the sketch in Fig. 1 encompasses both strategies. The reference signal source selection then provides the reference signal samples, $r^{(p)}[n]$, to be used for the current packet in the subsequent stages. 


\section{2) Modulation-dependent processing stage}

The processing scheme must typically include the clutter/multipath cancellation stage for the disturbance removal. Different approaches could be employed to implement this stage. In particular, the Extensive Cancellation Algorithm (ECA) and its modified versions have been shown especially effective aiming at mitigating the effect of the returns from the stationary scene while preserving slowly moving target echoes [37]. The ECAbased approaches operate by subtracting from the surveillance signal properly scaled and delayed replicas of the reference signal:

$$
\begin{gathered}
s_{0}^{(p)}[n]=s^{(p)}[n]-\sum_{k=0}^{K-1} \hat{\alpha}_{k}^{(p)} r^{(p)}[n-k] \\
n=0, \ldots, N_{s}(p)-1, p=0, \ldots, N_{P}-1
\end{gathered}
$$

The filter length $K$ is set according to the maximum expected delay for the multipath rays and its coefficients $\widehat{\boldsymbol{\alpha}}^{(p)}=$ $\left[\begin{array}{llll}\hat{\alpha}_{0}^{(p)} & \hat{\alpha}_{1}^{(p)} & \ldots & \hat{\alpha}_{K-1}^{(p)}\end{array}\right]^{T}$ are evaluated by resorting to a Least Square (LS) approach that minimizes the power of the signal at the output of the filter:

$$
\widehat{\boldsymbol{\alpha}}^{(p)}=\underset{\boldsymbol{\alpha}}{\operatorname{argmin}}\left\{\sum_{q \in I(p)} \sum_{n=0}^{N_{S}(p)-1}\left|s_{0}^{(q)}[n]\right|^{2}\right\}
$$

denoting $I(p)$ as the set of indices corresponding to the signal packets actually used for the estimation of the filter coefficients to be used at the $p$-th packet. The conventional ECA is obtained when $I(p) \equiv\left\{0, \ldots, N_{P}-1\right\}$, namely when all the $N_{P}$ packets of the considered processing interval are used. In contrast, the batch and the sliding version of the ECA, respectively referred to as ECA-B and ECA-S, could be obtained by using sub-sets of the available packets forming juxtaposed or overlapped batches, respectively [37]. The solution of (3) yields the following expression of the filter coefficients when a pulsed type transmission is exploited:

$$
\widehat{\boldsymbol{\alpha}}^{(p)}=\left[\widehat{\mathbf{M}}^{(p)}\right]^{-1} \widehat{\mathbf{v}}^{(p)}
$$

where $\widehat{\mathbf{M}}^{(p)}$ is a $K \times K$ matrix defined as

$$
\begin{gathered}
\widehat{\mathbf{M}}^{(p)} \equiv\left\{\widehat{m}_{l k}^{(p)}\right\}_{l, k=0, . ., K-1} \\
\hat{m}_{l k}^{(p)}=\sum_{q \in I(p)} \sum_{n=0}^{N_{S}(p)-1} r^{*(q)}[n-l] r^{(q)}[n-k]
\end{gathered}
$$

and $\widehat{\mathbf{v}}^{(p)}$ is a $K \times 1$ vector evaluated as

$$
\begin{gathered}
\hat{\mathbf{v}}^{(p)} \equiv\left\{\hat{v}_{l}^{(p)}\right\}_{l=0, . ., K-1} \\
\hat{v}_{l}^{(p)}=\sum_{q \in I(p)} \sum_{n=0}^{N_{S}(p)-1} r^{*}{ }^{(q)}[n-l] S^{(q)}[n]
\end{gathered}
$$

It is interesting to observe that, the filter coefficients are largely dependent on the autocorrelation characteristics of the employed signals and these should be averaged across packets sharing the same modulation scheme. In other words, the set of packets $\bar{I}(p)$ to be exploited for the estimation of $\widehat{\mathbf{M}}^{(p)}$ and $\hat{\mathbf{v}}^{(p)}$ should be actually limited to those transmitted using the same Standard for the physical layer, i.e., $\bar{I}(p)=$ $I(p) \cap J_{r}$, where $J_{r}$ is the set of indices corresponding to the signal packets transmitted using the $r$-th modulation scheme. This observation in turn explains the need for parallel processing chain to process different packets flows.

The signals packets at the output of (2) then undergo the range compression stage that evaluates the cross-correlation between the clutter cancelled surveillance signal and the reference signal on a packet-by-packet basis:

$$
\chi_{0}^{(p)}[l]=\sum_{n=0}^{N_{s}(p)-1} r^{*(p)}[n-l] s_{0}^{(p)}[n]
$$

If the reference signal is a perfect copy of the transmitted signal, this step basically implements a matched filter. A properly modified version $\tilde{r}^{(p)}[n]$ of the reference signal could be used instead in (7) with the aim to limit the sidelobes level of the signal AF in the delay dimension [17]. Notice that, the technique to be applied for the signal conditioning

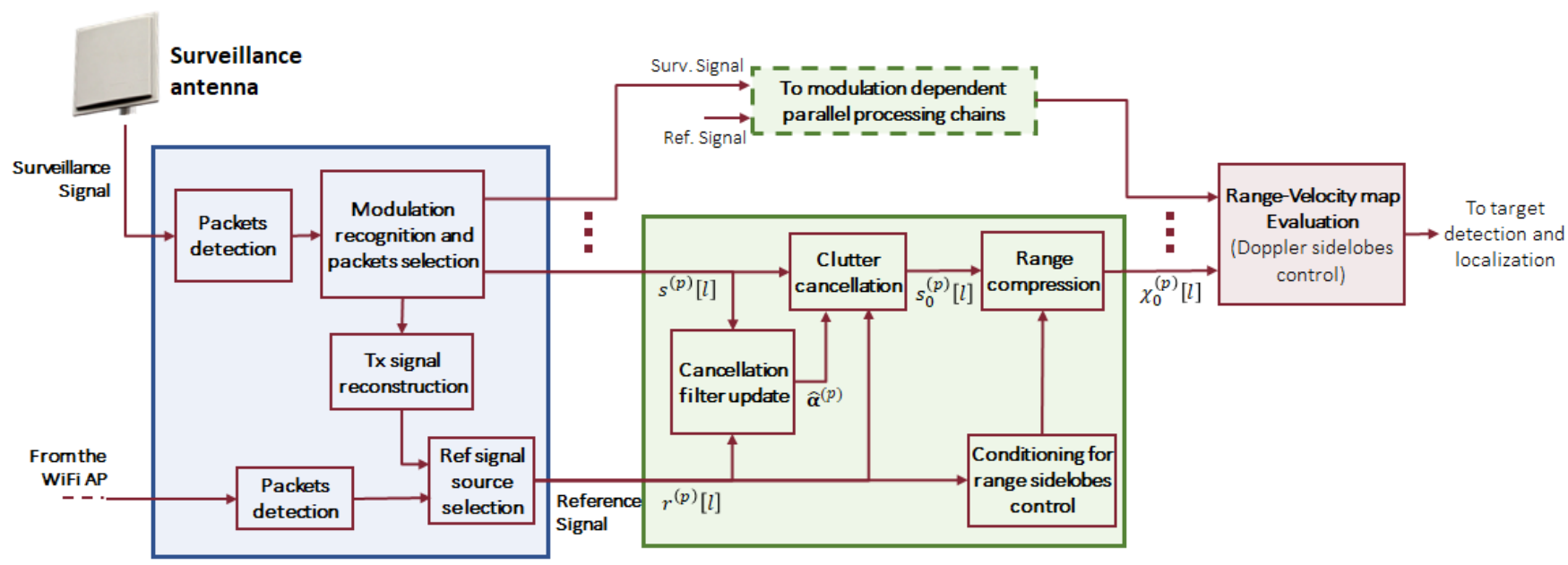

Pre-processing stage

Modulation dependent processing stage
Doppler processing stage

Fig. 1. Signal processing scheme for a WiFi-based passive radar exploiting mixed type transmissions 
depends on the adopted modulation scheme so that different approaches are implemented at the modulation-dependent parallel processing chains among those proposed in the technical literature [26][27].

\section{3) Doppler processing stage}

Subsequently, the results obtained for all the $N_{P}$ available packets within the CPI are coherently integrated according to a bank filters tuned to different target bistatic Doppler values defining a grid with spacing $\Delta f$, namely different slopes for the target motion induced linear phase law:

$$
\psi[l, m]=\sum_{p=0}^{N_{p}-1} e^{-j 2 \pi \frac{m \Delta f}{f_{s}} N_{0}(p)} \chi_{0}^{(p)}[l]
$$

This provides the final bistatic range-velocity map $\psi[l, m]$ to be exploited for target detection and localization. For packets of limited duration, this operation approximates the cross-ambiguity function between the reference signal and the surveillance signal at the output of the cancellation stage. This is certainly the case in WiFi sensing application against slowly moving targets, especially when exploiting transmission standards at carrier frequencies $f_{0}$ in the 2.4 $\mathrm{GHz}$ and $5 \mathrm{GHz}$ bands. In fact, with the exploited wavelength $\lambda$, the echo signal phase variation induced by typical target velocities $v_{b}$ (in the order of few $\mathrm{m} / \mathrm{s}$ ) within the packet duration $T_{P}(\ll 1 \mathrm{~ms})$ is largely negligible:

$$
\Delta \varphi=2 \pi \frac{v_{b}}{\lambda} T_{P} \ll 2 \pi,
$$

Again, proper tapering windows could be used in (8) for Doppler sidelobes control. Conventional windows can be verified to be ineffective against the Doppler sidelobes structures arising from a non-uniform sampling in the slow time as well as the amplitude modulation produced by the packets energy variation. To cope with this limitation, the WiFi packets available in the current CPI can be limited to a common length, i.e., an equal number $N_{S}$ of samples are processed at each packet via the previous stages; in the following, we will work under this assumption which is very easy to be guaranteed. Moreover, the adaptive sidelobes control technique presented in [29] can be exploited which is based on the solution of a constrained problem to identify a suitable weighting window.

The signal processing scheme described above has been shown to be effective in a number of scenarios where a WiFibased passive radar was tested for. Nevertheless, the computational complexity may limit its practical application, especially when a real-time processing is required. In particular, the most demanding processing blocks are those included in the modulation-dependent processing stage since they implement adaptive disturbance cancellation and range compression by operating against the raw signal samples across the whole processing interval. In contrast, the following Doppler processing stage takes as input the range compressed data that is produced at packet rate and the computation in (8) can be limited to the range bins of interest by discarding the samples of $\chi_{0}[l]$ for $l>l_{\max }$. Notice that a few range bins are typically included in short range surveillance applications.

The computational complexity of the modulationdependent processing stage could be lowered by observing that some operations performed in the cancellation and the range compression stages are in common and could be performed just once if the corresponding processing blocks are properly arranged.

To this purpose, by substituting (2) in (7), we rewrite the output of the range compression stage as:

$$
\chi_{0}^{(p)}[l]=\chi^{(p)}[l]-\sum_{k=0}^{K-1} \hat{\alpha}_{k}^{(p)} \chi_{r}^{(p)}[k-l]
$$

where

$$
\chi^{(p)}[l]=\sum_{n=0}^{N_{S}-1} r^{*(p)}[n-l] s^{(p)}[n]
$$

is the range compressed version of the input signal, and

$$
\begin{aligned}
\chi_{r}^{(p)}[l] & =\sum_{n=0}^{N_{S}-1} r^{*(p)}[n-l] r^{(p)}[n] \\
& \cong \sum_{n=0}^{N_{S}-1} r^{*(p)}[n-(l+k)] r^{(p)}[n-k]
\end{aligned}
$$

is the range compressed reference signal, namely the pointlike target response, and we have assumed that the exploited signal of opportunity has quasi-stationary characteristics. Therefore, based on (10), the output range compressed signal for the $p$-th packet is computed as a combination of the corresponding range compressed input signals.

In addition, we observe from eqs. (4)-(6) that the evaluation of the cancellation filter coefficients is based on the same range compressed signals which are then averaged across an appropriate number of packets. Specifically, using the definitions in (11)-(12), the elements of matrix $\widehat{\mathbf{M}}^{(p)}$ and vector $\hat{\mathbf{v}}^{(p)}$ in eqs. (5)-(6) can be easily expressed as:

$$
\widehat{m}_{l k}^{(p)}=\sum_{q \in I(p)} \chi_{r}^{(q)}[l-k]
$$

which yields a Toeplitz matrix $\widehat{\mathbf{M}}^{(p)}$, and

$$
\hat{v}_{l}^{(p)}=\sum_{q \in I(p)} \chi^{(q)}[l]
$$

Based on these considerations, the modulation-dependent processing stage can be modified as sketched in Fig. 2. According to the modified implementation, the range compression stage is first applied to both the surveillance and the reference signal on a packet-by-packet basis.

The corresponding outputs are then used for evaluating $\widehat{\mathbf{M}}^{(p)}$ and $\widehat{\mathbf{v}}^{(p)}$ and for updating the cancellation filter coefficients. Eventually, the clutter cancelled output is obtained by linearly combining the range compressed inputs with coefficients given by $\widehat{\boldsymbol{\alpha}}^{(p)}$. 


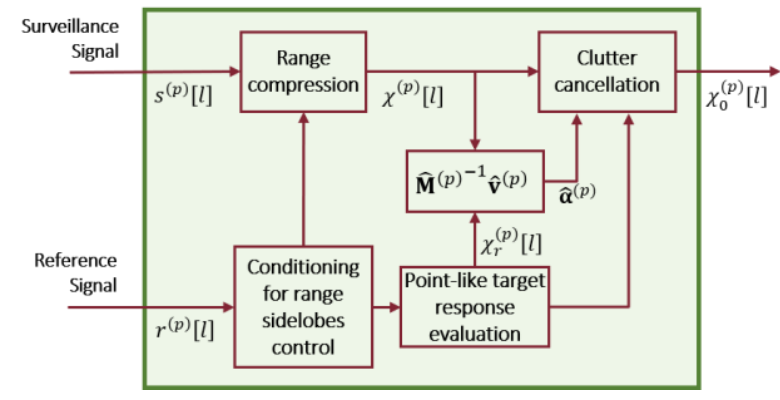

Fig. 2. Revised modulation-dependent processing stage.

Notice that this modified implementation reduces the computational cost since the most demanding operations, namely the range compression of the input signals, are only performed once. Clearly, the overall computational burden reduction will strictly depend on the employed parameters. Moreover, we observe that the output of the range compression stage could be truncated in the range dimension before being sent in input to the cancellation stage therefore significantly relaxing the data management requirements.

Despite these simplifications, the complexity of the overall signal processing architecture is still quite high due to the presence of modulation-dependent parallel processing chains. We recall that the dependency is inherent in the range sidelobes level control and in the adaptive estimation of the cancellation filter coefficients since the employed techniques exploit the auto-correlation properties of the signals of opportunity, which depend on the adopted modulation scheme.

Based on the considerations above, we investigate in the following the use of the $\mathrm{RpF}$ in lieu of the MF to operate the range compression stage as a mean to relax the dependency on the transmitted signals characteristics, thus allowing a further simplification of the signal processing architecture.

\section{RECIPROCAL FILTER BASED RANGE COMPRESSION} AND ITS IMPACT ON THE SIGNAL PROCESSING SCHEME

Let $S^{(p)}[m]$ and $R^{(p)}[m]$ denote the Discrete Fourier Transform (DFT) of the surveillance and reference signals at the $p$-th packet $\left(p=0, \ldots, N_{p}-1\right)$, respectively, i.e.

$$
\begin{aligned}
& S^{(p)}[m]=\operatorname{DFT}\left\{s^{(p)}[n]\right\} \\
& R^{(p)}[m]=\operatorname{DFT}\left\{r^{(p)}[n]\right\}
\end{aligned}
$$

Based on these definitions, the MF based range compression stage in (11) can be replaced by its RpF based version performed as

$$
\bar{\chi}^{(p)}[l]=I D F T\left\{\frac{S^{(p)}[m]}{R^{(p)}[m]} \cdot \operatorname{rect}_{\left.\mid \frac{B^{(p)}}{f_{S}} N_{S}\right]}[m]\right\}
$$

Note that, to avoid the issues due to overweighting possibly caused by the RpF, we limit its application to an appropriate bandwidth $B^{(p)}$, e.g., $B^{(O F D M)}=16.6 \mathrm{MHz}$ for OFDM packets and $B^{(D S S S)} \geq 11 \mathrm{MHz}$ for DSSS packets, forcing the output outside that band to be zero.

When this approach is applied against the reference signal, a rectangle-shaped output spectrum is obtained that takes non-zero values over the useful bandwidth $B^{(p)}$. Therefore, the point-like target response provided by the $\mathrm{RpF}$ at the $p$-th packet can be evaluated as:

$$
\begin{aligned}
\bar{\chi}_{r}^{(p)}[l] & =\operatorname{IDFT}\left\{\frac{R^{(p)}[m]}{R^{(p)}[m]} \cdot \operatorname{rect}_{\left.\left.\mid \frac{B}{f_{s}} N_{s}\right\rfloor^{[m]}\right\}}\right. \\
& =\frac{\sin \left(\pi \frac{l}{N_{s}}\left\lfloor\frac{B^{(p)}}{f_{s}} N_{s}\right\rfloor\right)}{\sin \left(\pi \frac{l}{N_{s}}\right)}=\eta\left[l ; B^{(p)}\right]
\end{aligned}
$$

Eq. (17) clearly shows that the point-like target response of the RpF at the $p$-th packet $\bar{\chi}_{r}^{(p)}[l]$ is independent of the transmitted signal, i.e., it does not change with the information content. The dependence on the modulation scheme adopted at $p$-th packet is limited to the extent of the useful bandwidth $B^{(p)}$. Therefore, $\bar{\chi}_{r}^{(p)}[l]$ can be easily computed based on the well-known $d \operatorname{sinc}$ function $\eta\left[l ; B^{(p)}\right]$.

The considerations above have significant consequences on the overall signal processing scheme as well as on the achievable performance, as discussed in the following subsections that separately address different aspects.

\section{A. Range sidelobes control}

Eq. (17) shows that, when the RpF is applied, we deal with a rectangle-shaped output spectrum, regardless of the employed modulation. This implies that an effective sidelobe control (SLC) in the range domain can be easily obtained with conventional tapering windows (e.g., Hamming), without requiring the design and implementation of ad hoc approaches. Notice that the SLC does not alter the good properties observed in (17) since the tapering simply yields:

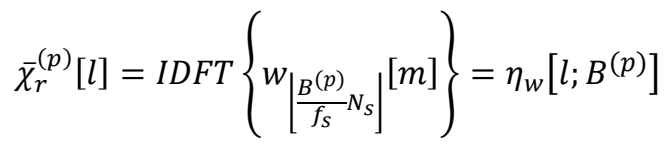

where $w_{M}[m]$ is the selected window and the dependence on the modulation scheme adopted at $p$-th packet is again limited to the band extent where the window is applied.

Instead, it is worth recalling that, when the MF is used, the tapering window must be properly adapted to the employed modulation. For instance, when DSSS modulated packets are received, an ad hoc tapering window is needed to account for the sidelobe structures due to the employed 11chip Barker code. To this purpose, in [18] a Barker Weighting network (BWN) was derived as the result of a constrained optimization problem which starts from the knowledge of the Barker code auto-correlation function (ACF). Additionally, in [18] an adaptive filter is proposed aiming at counteracting the additional sidelobes depending on the average correlation among consecutive symbols. In contrast, when using OFDM transmissions, conventional tapering windows have been exploited [27][28]. However, their effectiveness has been shown to be limited due to the non-perfectly flat signal spectrum.

The effectiveness of a conventional tapering window when the RpF is employed is shown in Fig. 3(a-b) where we compare the point-like target response obtained with the $\mathrm{RpF}$ and the MF for a given WiFi packet, when DSSS and OFDM modulations schemes are adopted, respectively. 


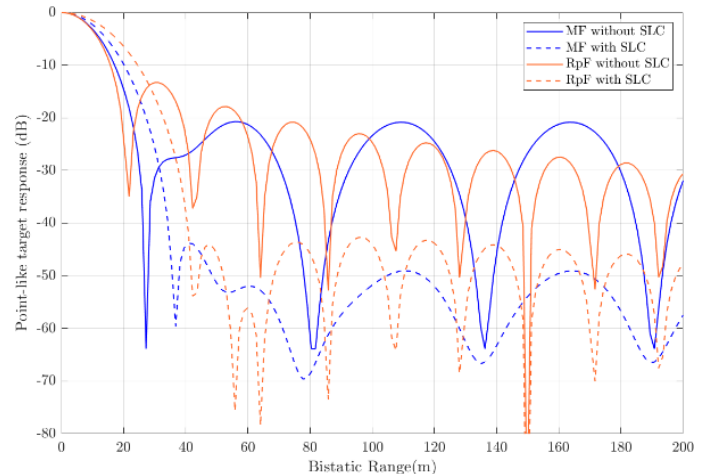

(a)

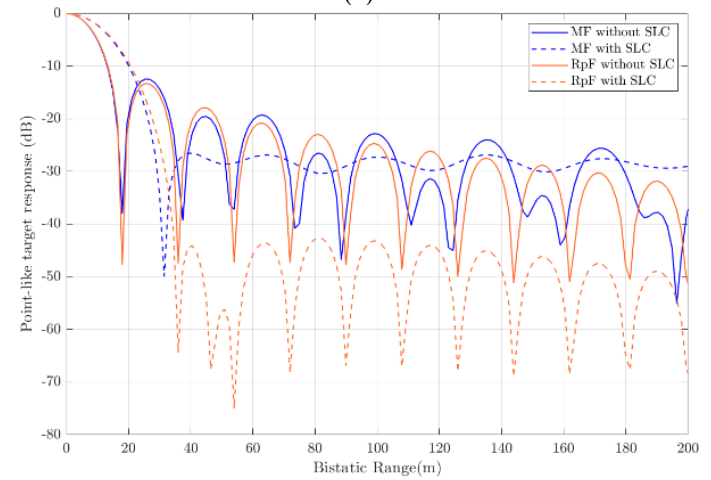

(b)

Fig. 3. Point-like target response using either the MF of the RpF for range compression, with and without SLC for (a) DSSS modulated packet (b) OFDM modulated packet.

In both sub-figures, the blue lines represent the results obtained with the MF while the orange lines are for the RpF. Continuous lines are for the results without any SLC approach while dashed lines are obtained when the range sidelobe level is controlled with the aforementioned weighting functions. All curves are normalized to their maximum peak in order to focus on the obtained sidelobe level and the resolution degradation.

Based on Fig. 3 we confirm that:

(i) For DSSS packets, an effective SLC is obtained when the MF is used for range compression and the tapering window is properly optimized (blue curves in Fig. 3(a)).

(ii) For OFDM packets, when the MF is applied for range compression, the limited effectiveness of the conventional Hamming function is apparent (blue curves in Fig. 3(b)). This suggests the need for ad hoc solutions as for the DSSS case, which however require a dedicated design.

(iii) When the $\mathrm{RpF}$ is considered, a conventional tapering window (e.g., the Hamming function) is a simple and effective solution for both the DSSS and OFDM packets, yielding a comparable SLC as the MF for the DSSS case while further reducing the sidelobe level of approx. $20 \mathrm{~dB}$ in the OFDM case. The slight resolution degradation in the DSSS case is due to the application of the RpF over a bandwidth of $B^{(D S S S)}=14 \mathrm{MHz}$, which implies filtering out the signal spectrum tails.

\section{B. Clutter removal}

After the range compression stage has been performed using the RpF approach, the output can undergo the clutter cancellation stage as described in Section II. According to the
ECA approach, the output of this stage is obtained as in (10), but replacing $\chi_{r}^{(p)}$ and $\chi^{(p)}$ with their counterparts at the output of the RpF. Similarly, the cancellation filter coefficients $\widehat{\boldsymbol{\alpha}}^{(p)}$ are obtained using (4), with $\widehat{\mathbf{M}}^{(p)}$ and $\widehat{\mathbf{v}}^{(p)}$ estimated as in (13)-(14) where $\chi_{r}^{(p)}$ and $\chi^{(p)}$ are replaced by $\bar{\chi}_{r}{ }^{(p)}[l]$ and $\bar{\chi}^{(p)}[l]$, respectively.

However, the result in (17) suggests the possibility of a simplified clutter cancellation stage that leverages the dataindependent characteristics of $\bar{\chi}_{r}^{(p)}[l]$. In fact, using (17), eq. (10) can be simplified as

$$
\bar{\chi}_{0}^{(p)}[l]=\bar{\chi}^{(p)}[l]-\sum_{k=0}^{K-1} \hat{\alpha}_{k}^{(p)} \eta_{w}\left[l-k ; B^{(X)}\right]
$$

where $B^{(X)}$ is either $B^{(O F D M)}$ or $B^{(D S S S)}$ depending on the parallel processing chain considered and the coefficients $\widehat{\boldsymbol{\alpha}}^{(p)}$ are used to build up a linear combination of known functions. Moreover, these coefficients are evaluated using an a priori computed matrix $\mathbf{M}^{(p)}=\mathbf{M}$ whose generic element is given by

$$
m_{l k}^{(p)}=m_{l k}=P \eta_{w}\left[l-k ; B^{(X)}\right]
$$

where $P$ is the cardinality of set $I(p), \forall p$.

In other words, the estimation and the inversion of matrix $\mathbf{M}$ are no longer needed and the estimation of the cancellation filter coefficients to be applied at the $p$-th packet can be simplified using the clairvoyant version of $\mathbf{M}$ :

$$
\widehat{\boldsymbol{\alpha}}^{(p)}=\mathbf{M}^{-1} \hat{\mathbf{v}}^{(p)}
$$

In the following, this approach will be referred to as ECAa priori and can be regarded as a direct consequence of the equalization of the transmitted signal spectrum yield by the $\mathrm{RpF}$. Accordingly, the processing block in Fig. 2 simplifies as in Fig. 4. Note that the complexity reduction obtained with the ECA-a priori is particularly evident when the estimation and inversion of the matrix needs to be repeated along the CPI, e.g., using ECA-B or ECA-S [37].

To prove the effectiveness of the ECA- $a$ priori, we consider a simulated scenario including the direct signal from the AP, with input direct signal-to-noise ratio (DNR) of 40 $\mathrm{dB}$, thermal noise, and several multipath contributions with delays (with respect to the direct path) between $1.5 \mathrm{~ns}$ and $82.6 \mathrm{~ns}$ and SNR levels between $-13 \mathrm{~dB}$ and $36 \mathrm{~dB}$, depending on the distance traveled. Additional details on the simulated scenario are reported in Table 1.

First, in Fig. 5, we show the output of the range-velocity map for a CPI of $0.5 \mathrm{~s}$ with SLC but when the cancellation is not applied, using the conventional MF (a) or the RpF (b).

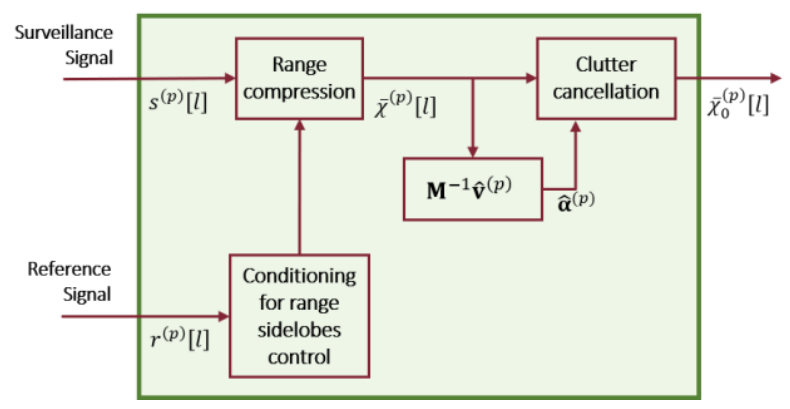

Fig. 4. Revised modulation-dependent processing stage with ECA-a priori. 
In this case, a WiFi transmission including only OFDM modulated packets is used. Both maps have been scaled for the output noise level to be around $0 \mathrm{~dB}$. In both cases, the stationary contributions of both the direct signal and the strongest multipath rays are evident. However, when applying the $\mathrm{RpF}$, the map appears generally darker since (i) the SLC is more effective, and (ii) the RpF strategy is expected to yield an SNR loss compared to the MF. However, this also suggests that, when the $\mathrm{RpF}$ is applied in low clutter scenarios, a cancellation stage might not be strictly necessary to discriminate targets that do not lie around the zero-Doppler axis.

Table 1 Employed Simulation Parameters.

\begin{tabular}{|cc|}
\hline Parameter & Value \\
\hline Carrier frequency $\left(f_{0}\right)$ & $2.4 \mathrm{GHz}$ \\
Wavelength $(\lambda)$ & $0.1249 \mathrm{~m}$ \\
Sampling frequency $\left(f_{\mathrm{s}}\right)$ & $20 \mathrm{MHz}$ \\
Modulation & OFDM/DSSS \\
Pulse repetition interval (PRI) & $3 \mathrm{~ms}$ \\
Packet length & $0.16 \mathrm{~ms}$ \\
\hline
\end{tabular}

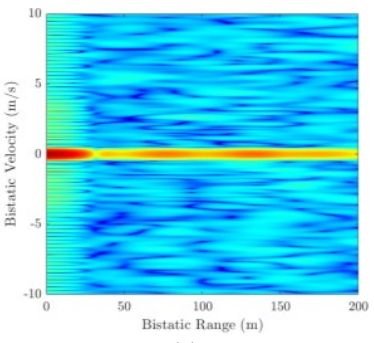

(a)

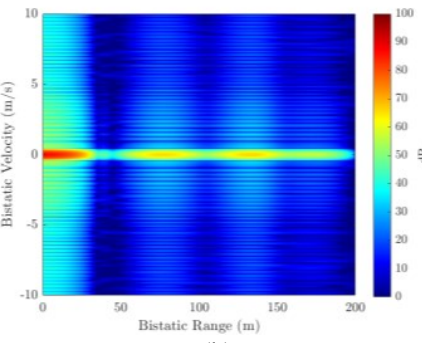

(b)
Fig. 5. Range-Velocity map without disturbance cancellation using MF (a) or $\mathrm{RpF}$ (b) for range compression, including SLC.

In contrast, in highly cluttered scenarios, the stationary disturbance cancellation is an essential stage for both $\mathrm{MF}$ based and $\mathrm{RpF}$ based processing schemes. The results of the application of the clutter removal stage are reported in Fig. 6 for the considered case study. As a first result, we investigate the use the ECA when applied after the MF (Fig. 6(a)) and the RpF (Fig. 6(b)), showing that it keeps its effectiveness whichever the technique adopted for range compression is. This is not the case for the ECA- $a$ priori which fails at removing the clutter returns when the MF is used (Fig. 6(c)) since the simplifications discussed in this sub-Section do not apply. Incidentally, note that this result is obtained with OFDM transmissions, which, among the typical WiFi signal modulations, present the flattest spectral shape. Therefore, even higher background levels are expected when the cascade of the MF and the ECA- $a$ priori approach are applied to DSSS modulated packets. In contrast ECA- $a$ priori provides remarkable performance when applied after the RpF (Fig. 6(d)); as expected, in this case, the simplified ECA version and the conventional ECA yield identical outputs. This result has been shown to hold also when different modulation schemes are considered thanks to the modulationindependent characteristics of the RpF output.

\section{Mixed modulation transmissions}

Based on sub-sections III.A and III.B, a modified signal processing scheme has been obtained which requires identical strategies for SLC and clutter cancellation regardless of the adopted modulation. Still, the capability to recognize the packets modulation and the subsequent parallel processing chains might be required if the above scheme is applied against different signal bandwidths, tailored for OFDM and DSSS packets.

Therefore, an effective way to further simplify the overall processing scheme when the $\mathrm{RpF}$ is used is to filter the packet bandwidth to a common value $B$, which yields the block diagram shown in Fig. 7. Note that, depending on the expected packet stream, the value of $B$ might change. If the system expects that the majority of the collected packets are with DSSS modulation, one might consider using $B=$ $B^{(D S S S)}$. However, the predominance of OFDM packets in modern WiFi systems makes a $16.6 \mathrm{MHz}$ cutoff more convenient, aiming at also preserving the range resolution. Based on this consideration, in the following, when referring to a unified processing scheme with a common bandwidth, we set $B=B^{(O F D M)}=16.6 \mathrm{MHz}$.

First, we investigate the effectiveness of the proposed unified processing scheme of Fig. 7 in terms of SLC. To this purpose we consider a reasonable configuration in terms of mixing ratio between OFDM and DSSS packets appearing within the CPI, i.e., $80 \%$ OFDM packets - 20\% DSSS packets. Basically, in the considered mixed transmission case, we admit the presence of users with different equipment operating according to different standards. In contrast, the case of $100 \%$ OFDM packets considered in the previous subsections is representative of a modern WiFi system where the presence of DSSS modulated packets is only due to beacons, transmitted with a very large beacon interval.

The point-like target response for mixed transmissions is reported in Fig. 8 using different strategies. As already noted in Fig. 3(b), the capability to obtain a proper SLC is jeopardized when the MF based signal processing chain is applied in conjunction with a conventional Hamming tapering window. This is overall confirmed here where the OFDM modulated packets represent most, although not all,

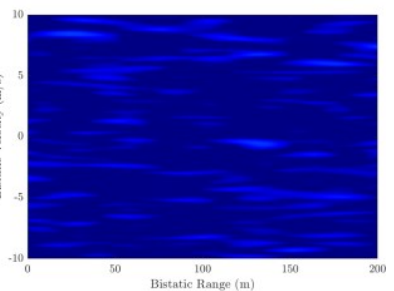

(a)

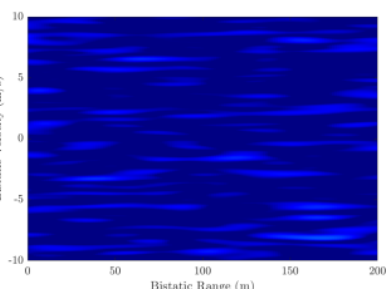

(b)

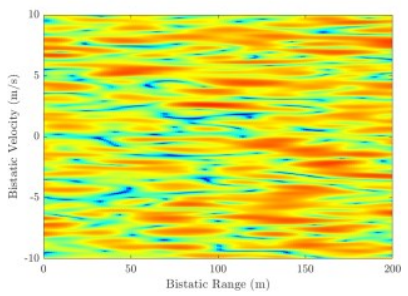

(c)

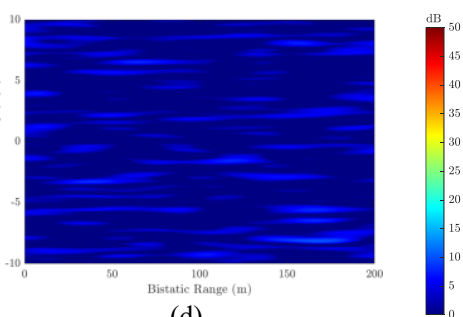

(d)

Fig. 6. Range-Velocity map for the simulated scenario:

(a) MF with ECA (b) RpF with ECA (c) MF with ECA-a priori (c) RpF with ECA-a priori 


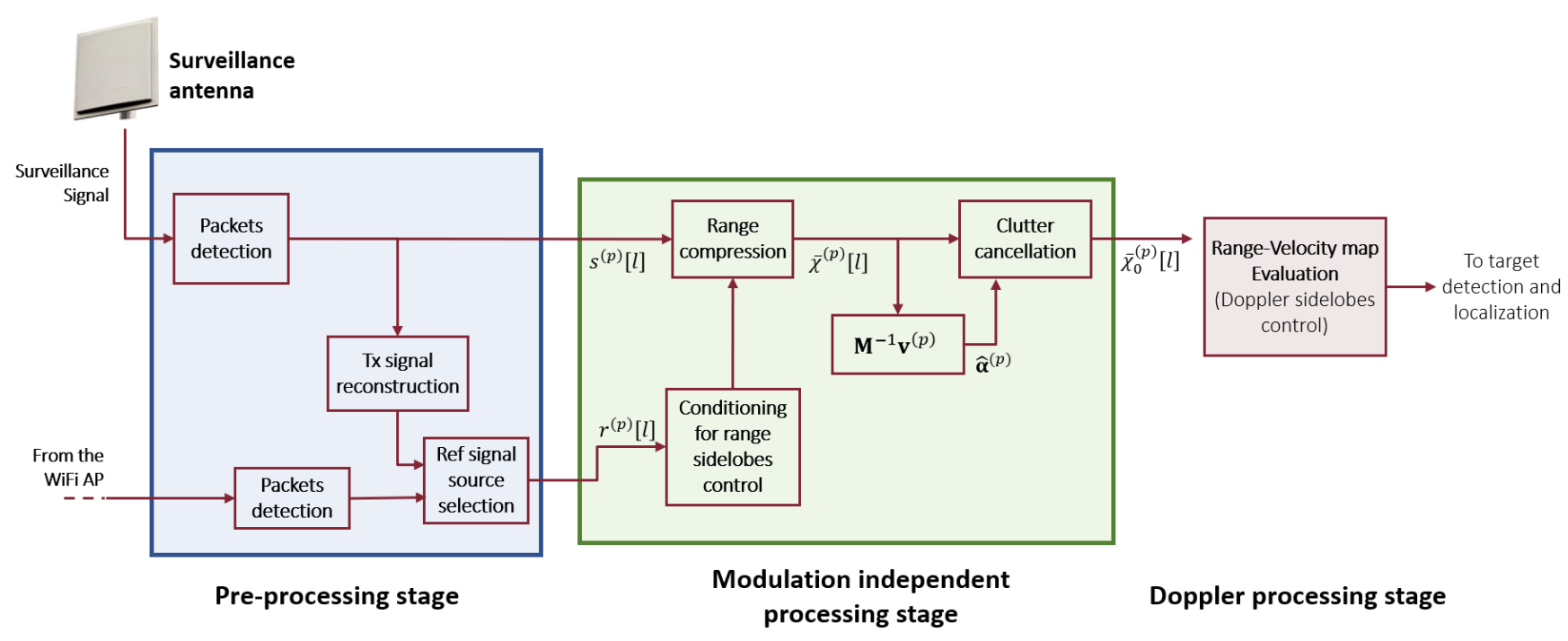

Fig. 7. RpF based signal processing scheme for a WiFi-based passive radar with unified SLC and ECA-a priori.

of the employed packets.

In fact, comparing the dashed blue lines in Fig. 3(b) and Fig. 8, namely increasing the number of DSSS packets, the obtained sidelobe level only slightly decreases. Similarly, using a unified MF based scheme, namely treating all packets as if they were OFDM modulated, does not yield a significant SLC degradation (compare the dashed and the dashed-dot blue lines).

Moreover, as stated above, the RpF based scheme allows an effective SLC, regardless of it not being adapted to the packet bandwidth. Comparable results are obtained with a modulation dependent RpF strategy (dashed orange curve), recalling that the dependence only lies in the employed bandwidth.

Finally, comparing the sidelobe level obtained with the modulation dependent MF based strategy and the unified RpF based scheme, the latter yields an improvement of approx. 20 $\mathrm{dB}$. This would enable the detection of small RCS targets that might be buried below the range sidelobes in the first case.

Now that the range SLC capability of the unified RpF based processing has been demonstrated, it is worth investigating the robustness of the proposed ECA- a priori for clutter cancellation, also when mixed modulated transmissions are employed.

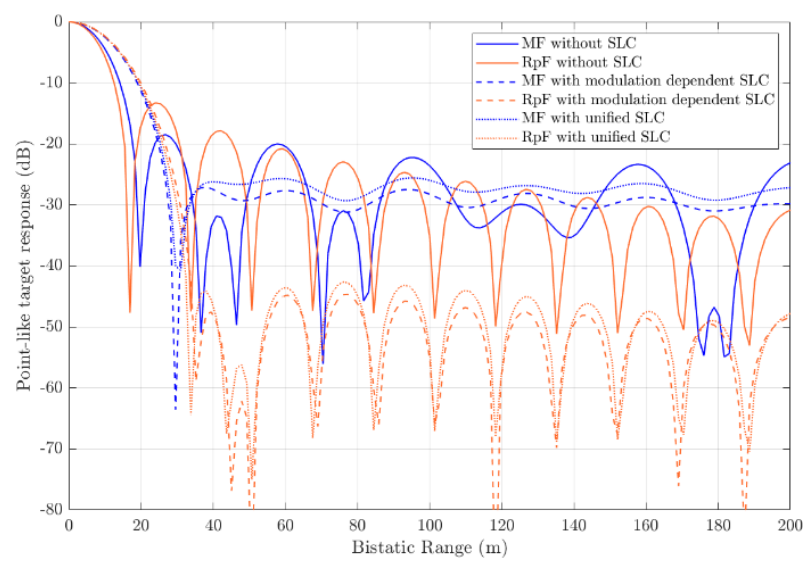

Fig. 8. Point-like target response using different approaches for range compression and SLC with mixed modulation trasnmission.
To this end, we consider the case scenario simulated for Section III.A and we add four point-like moving targets with different input SNR $\left(\mathrm{SNR}_{\mathrm{I}}\right)$ and at different delays to emulate a short-range surveillance application. Details on the considered targets are reported in Table 2, where we report their positions in the bistatic range-velocity plane as well as the $\mathrm{SNR}_{\mathrm{I}}$ values. In Fig. 9 we analyze the two different mixing configurations and we compare the obtained rangevelocity maps when the modulation dependent MF based scheme (a-b) or the unified RpF based scheme (c-d) are applied. All maps are scaled for the output noise level to be around $0 \mathrm{~dB}$ so that the target peaks correspond to the output SNR, reported next to each peak for convenience.

The following considerations are in order:

- When the RpF based unified processing scheme is used (see Fig. 9 (c-d)), the clutter cancellation block can be simplified with the ECA-a priori approach, that preserves the disturbance rejection capability even when mixed modulated transmissions are exploited. In order to achieve similar results, the modulation dependent MF based scheme (see Fig. 9 (a-b)) requires the conventional ECA to be used with filter coefficients separately adapted at each processing chain. This is confirmed by Fig. 10(a-b) where the ECA- $a$ priori is used after the MF based range compression for the two different modulation mixing, using a unified SLC.

Table 2 Simulated target characteristics

\begin{tabular}{|c|c|c|}
\hline & Parameter & Value \\
\hline \multirow{3}{*}{$\stackrel{b_{0}}{\oplus} \#$} & Bistatic Range & $22.53 \mathrm{~m}$ \\
\hline & Bistatic Velocity & $1 \mathrm{~m} / \mathrm{s}$ \\
\hline & Input signal-to-noise ratio $\left(\mathrm{SNR}_{\mathrm{I}}\right)$ & $0 \mathrm{~dB}$ \\
\hline \multirow{3}{*}{$\stackrel{50}{\oplus} \cong$} & Bistatic Range & $92.96 \mathrm{~m}$ \\
\hline & Bistatic Velocity & $-2.3 \mathrm{~m} / \mathrm{s}$ \\
\hline & Input signal-to-noise ratio $\left(\mathrm{SNR}_{\mathrm{I}}\right)$ & $-10 \mathrm{~dB}$ \\
\hline \multirow{3}{*}{$\stackrel{50}{\oplus} \#$} & Bistatic Range & $122.5 \mathrm{~m}$ \\
\hline & Bistatic Velocity & $1.1 \mathrm{~m} / \mathrm{s}$ \\
\hline & Input signal-to-noise ratio $\left(\mathrm{SNR}_{\mathrm{I}}\right)$ & $-28 \mathrm{~dB}$ \\
\hline \multirow{3}{*}{$\stackrel{b_{0}}{\oplus}$} & Bistatic Range & $164.8 \mathrm{~m}$ \\
\hline & Bistatic Velocity & $7.06 \mathrm{~m} / \mathrm{s}$ \\
\hline & Input signal-to-noise ratio $\left(\mathrm{SNR}_{\mathrm{I}}\right)$ & $-30 \mathrm{~dB}$ \\
\hline
\end{tabular}




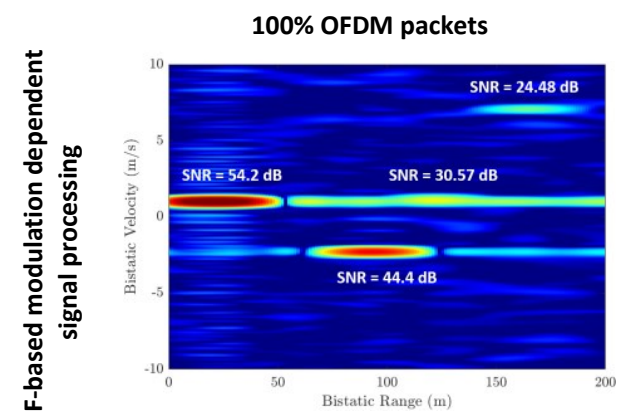

(a)

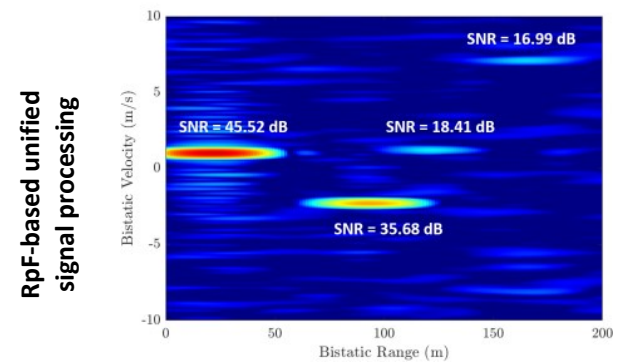

(c)
$80 \%$ OFDM - $20 \%$ DSSS packets

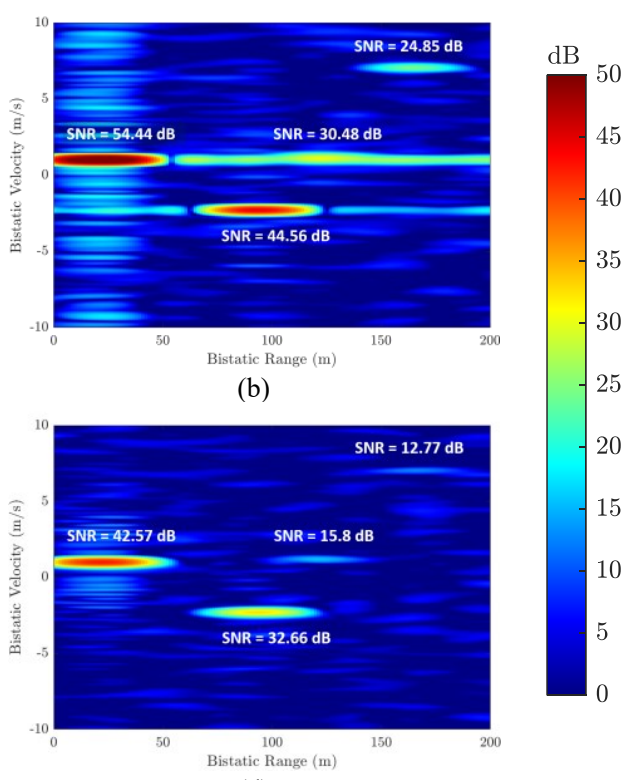

(d)

Fig. 9. Output range-velocity map for the simulated scenario when applying either the MF-based modulation dependent signal processing scheme or the RpF-based unified signal processing scheme to different packets configurations.

- When the modulation dependent MF based signal processing scheme is applied (see Fig. 9 (a-b)), the reduced SLC capability might cause the weak target echoes to be masked by the high sidelobes of the strongest targets. For instance, in the considered case study, target \#3 is buried in the sidelobes of target \#1. In contrast, the robust range SLC of the unified $\mathrm{RpF}$ based strategy results in the possibility to discriminate the different target contributions.

- As expected, all targets suffer from an SNR loss when the $\mathrm{RpF}$ is applied for range compression in lieu of the MF. In this case, the loss is between 7.5 and $12 \mathrm{~dB}$. Such loss might not be an issue in clutter-limited scenarios while it might jeopardize the detection of small RCS targets in noise-limited scenarios. The next section will be devoted to tackle this issue.

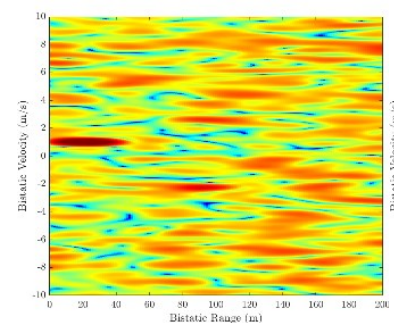

(a)

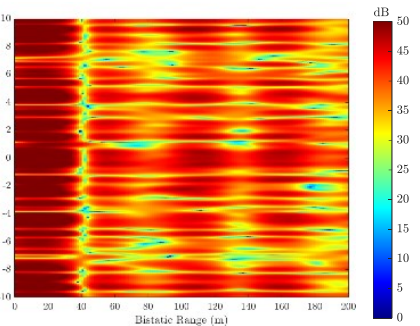

(b)
Fig. 10. Range-velocity map using the MF-based scheme with unified SLC and ECA-a priori, for: (a) 100\% OFDM packets (b) $80 \%$ OFDM $-20 \%$ DSSS packets.

\section{SUPERVISED RECIPROCAL FILTERING STRATEGIES}

We have shown in Section III that the application of the $\mathrm{RpF}$ for the range compression stage yields a non-negligible target SNR loss. This might become an issue when aiming at detecting very weak target echoes in noise-limited scenarios.

The SNR loss of the RpF has been theoretically evaluated in [33]-[35] when operating at OFDM symbol level and, in that case, it is expected to be in the order of few $\mathrm{dB}$. The increased loss observed with the proposed approach that operates against the entire WiFi packet can be explained by observing the deep notches that appear in each packet spectrum $R^{(p)}[m]$ (an example is reported in Fig. 11 for an OFDM packet scaled to its mean square value). When such spectrum is inverted to build the $\mathrm{RpF}$, these notches produce spikes that determine a significant amplification of the noise level thus reducing the resulting target SNR. The effect is larger when longer packets are exploited since that implies a larger oversampling in the frequency domain.

Based on these considerations, an intuitive approach to mitigate the observed SNR loss, is to introduce an appropriate supervision strategy to keep the impact of the deep spectral notches under control. In this Section, we tackle this issue by considering two possible simple approaches.

Zeros - Reciprocal Filter (Z-RpF): According to this strategy, the output of the $\mathrm{RpF}$ at the frequencies that trigger a selected threshold $\zeta$ is forced to be zero. Specifically, let us denote with $I_{\zeta}^{(p)}$ the corresponding set of frequency values:

$$
I_{\zeta}^{(p)} \equiv\left\{\left.m|| R^{(p)}[m]\right|^{2}<\zeta P^{(p)}\right\}
$$

$P^{(p)}$ being the mean power level of the reference signal spectrum at the $p$-th packet, measured within the useful bandwidth $B^{(p)}$. Eq. (16) is modified as:

$$
\bar{\chi}^{(p)}[l]=\operatorname{IDFT}\left\{\mu^{(p)}[m]\right\}
$$

where

$$
\mu^{(p)}[m]= \begin{cases}\frac{S^{(p)}[m]}{R^{(p)}[m]} \cdot \operatorname{rect}_{\left.\mid \frac{B^{(p)}}{f_{S}} N_{S}\right]^{[m]}} & m \notin I_{\zeta}^{(p)} \\ 0 & m \in I_{\zeta}^{(p)}\end{cases}
$$




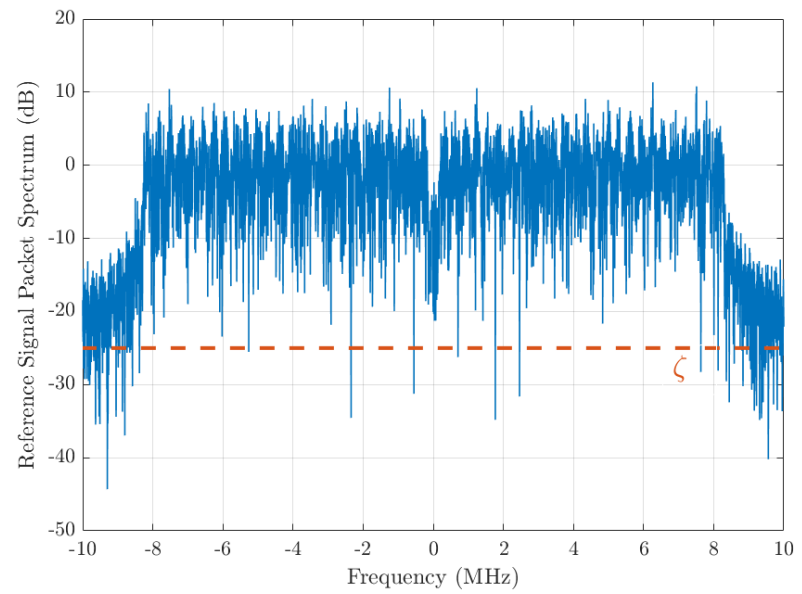

Fig. 11. Reference signal spectrum for OFDM modulated packet.

Interpolated - Reciprocal Filter (I-RpF): In this case, the frequencies that trigger a selected threshold $\zeta$ are excluded from the reciprocal filter evaluation. The value of the output spectrum at those frequencies is instead obtained via interpolation of the results at adjacent frequencies. In other words, eq. (23) holds with

$$
\mu^{(p)}[m]= \begin{cases}\frac{S^{(p)}[m]}{R^{(p)}[m]} \cdot \operatorname{rect}_{\left.\mid \frac{B^{(p)}}{f_{s}} N_{S}\right]}[m] & m \notin I_{\zeta}^{(p)} \\ F\left\{\mu^{(p)}[m] ; m \notin I_{\zeta}^{(p)}\right\} & m \in I_{\zeta}^{(p)}\end{cases}
$$

where $F\left\{x_{1}, x_{2}, \ldots\right\}$ indicates the selected interpolating function. A straightforward linear interpolation strategy can be used when long WiFi packets are exploited, namely when the corresponding spectra are largely oversampled, and moderate thresholding is applied so that only a few isolated samples are excluded (see for example Fig. 11). Obviously, depending on the complexity of the scenario of interest, alternative interpolation approaches could be considered.

In the following, the performance of these two supervised strategies is extensively studied as a function of the employed threshold with the purpose of investigating the robustness of the simplified processing scheme, presented in Section III, to the proposed modifications.

First, the SNR loss is analyzed, measured with respect to the conventional MF based processing scheme described in Section II operating without the additional loss due to the SLC stage. The results are evaluated for an OFDM only transmission, averaging the obtained values over 10 consecutive CPIs, and are reported in Fig. 12(a) across a nonuniformly sampled grid of threshold values between $-40 \mathrm{~dB}$ and $5 \mathrm{~dB}$. Specifically, the SNR loss curves are shown for the unified $\mathrm{RpF}$ based processing scheme with the two supervision strategies, with the application of the SLC stage. Additionally, the value obtained with the conventional MF based processing scheme when the SLC is applied is reported for comparison.

The SNR loss curves obtained when the supervision strategies are applied follow the same behavior. Specifically, there is an initial decay as the threshold increases. Then, both curves reach a minimum and the SNR loss starts increasing again for higher $\zeta$ values. The threshold value where the minimum SNR loss is obtained changes with the employed strategy. The Z-RpF strategy yields a minimum SNR loss at approx. $\zeta=-2 \mathrm{~dB}$ while the I-RpF strategy curve reaches is minimum at approx. $\zeta=-10 \mathrm{~dB}$. The minimum SNR losses measured with respect to the MF based processing with SLC (blue line) are equal to approx. $1 \mathrm{~dB}$ and $4 \mathrm{~dB}$ when the $\mathrm{Z}$ $\mathrm{RpF}$ and the I-RpF strategies are applied, respectively.

The results of Fig. 12(a) show that, by properly selecting the threshold, the SNR loss yield by the RpF can be largely reduced with any of the two considered approaches. However, since the implementation of these strategies inherently implies significant distortions to affect the output of the range compression stage, especially with the Z-RpF strategy, it is expected that the use of high threshold values might jeopardize the good characteristics of the $\mathrm{RpF}$, which allowed tremendous simplification of the WiFi-based passive radar processing scheme. Therefore, the threshold selection should also take into account the capability of preserving the results of Section III, both in terms of SLC and in terms of cancellation stage requirements.

To this purpose, the cancellation capability is investigated in Fig. 12(b) where we report the average background level (above noise) measured on the output range-velocity map, under the $H_{0}$ (target absent) hypothesis, when different processing schemes are used. The curves obtained with the two supervised RpF strategies are shown as a function of the $\zeta$, using either the ECA-a priori or the conventional ECA. As a reference, the values obtained with the MF-based processing schemes are also reported.

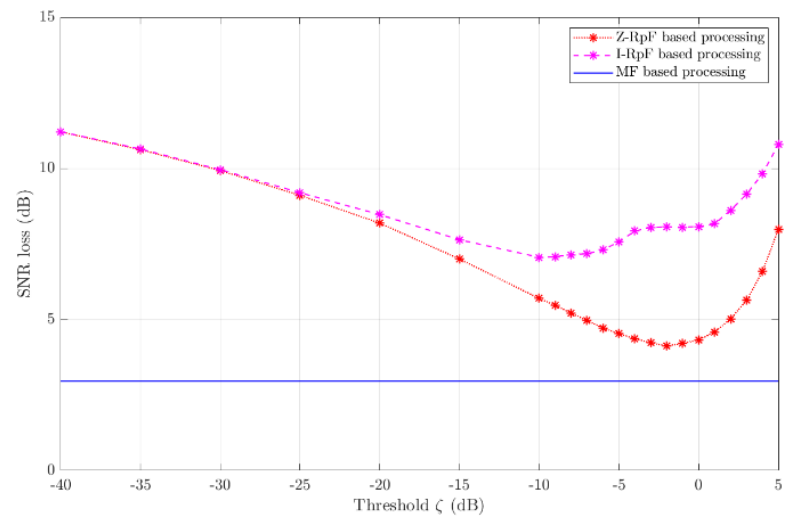

(a)

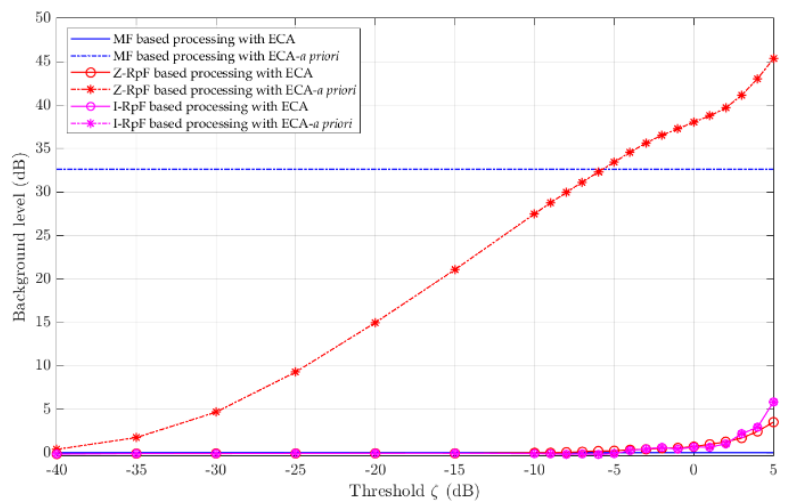

(b)

Fig. 12. Performance of the Z-RpF and I-RpF versus threshold for OFDMonly config: (a) SNR loss with respect to the MF strategy (b) measured background level. 
As in Fig. 12(a), the results are evaluated for an OFDM only transmission, and averaged over 10 consecutive CPIs.

First of all, we note that, with the conventional MF based processing scheme employing the ECA (continuous blue line), the background level on the map after cancellation is around $0 \mathrm{~dB}$, denoting an effective clutter removal. However, if the ECA-a priori is applied within the MF based processing scheme, the clutter cancellation is not at all effective and the measured background level is around $35 \mathrm{~dB}$ in the considered case study. Note that the dash-dot blue line in Fig. 12(b) confirms what shown in the range-velocity map in Fig. 6(c).

Similarly, when the Z-RpF strategy is applied, the background level after cancellation is kept under control only when the ECA is applied. On the contrary, using the Z-RpF with the ECA-a priori, the background level remarkably grows with $\zeta$ since the insertion of an increasing number of zeros jeopardizes the assumption of a flat output spectrum. Following the same considerations, the capability of controlling the range sidelobes is also expected to decrease.

When the I-RpF strategy is applied, an effective clutter cancellation is obtained, both when the ECA and the ECA- $a$ priori are used, up to very high values of the threshold $(\zeta<$ $0 \mathrm{~dB}$ ). This is due to the increased robustness of the spectral interpolation approach which does not significantly alter the characteristics of the original spectrum. For this reason, also the SLC capability is better preserved. A degradation is observed at higher values of the threshold due to the progressive reduction of the spectral samples available to obtain a meaningful interpolation.

By combining the metrics in Fig. 12(a) and (b), we built Fig. 13(a), which reports the performance of the different approaches in terms of output signal-to-clutter plus noise ratio (SCNR) for a reference target whose expected maximum output SNR is equal to $\left.\sigma\right|_{\mathrm{dB}}=20 \mathrm{~dB}$ when an ideal MF is applied without SLC. Specifically, each curve is obtained as $\left.\mathrm{SCNR}\right|_{\mathrm{dB}}=\left.\sigma\right|_{\mathrm{dB}}-$ Loss $\left.\right|_{\mathrm{dB}}-\left.\mathrm{BL}\right|_{\mathrm{dB}}$, where Loss $\left.\right|_{\mathrm{dB}}$ and $\left.\mathrm{BL}\right|_{\mathrm{dB}}$ are the SNR loss and the background level in decibel. Similarly, Fig. 13(b) shows the SCNR curves obtained when a mixed modulation transmission is employed using one of the mixing configurations previously considered, i.e., $80 \%$ OFDM packets $-20 \%$ DSSS packets.

These figures confirm what already noted separately observing Fig. 12(a) and (b) and prove that the proposed supervised $\mathrm{RpF}$ strategies are effective also when applied against packets exploiting different modulations.

More precisely, in all cases, the Z-RpF based processing scheme yields the highest SCNR among the RpF based strategy but only if followed by a conventional ECA for clutter removal. As for the MF based processing scheme, due to the high background level, the use of the ECA- $a$ priori after the Z-RpF yields unacceptable SCNR values. However, we note that the processing scheme encompassing the $\mathrm{Z}-\mathrm{RpF}$ and the ECA still guarantees a reduced complexity with respect to the conventional MF based scheme since a single (modulation-independent) processing chain is required.

When the I-RpF based processing is considered and the threshold is properly selected, the output SCNR is only 4-5 $\mathrm{dB}$ less than that obtained with the MF based scheme for both ECA and ECA-a priori in both cases (Fig. 13(a-b)).

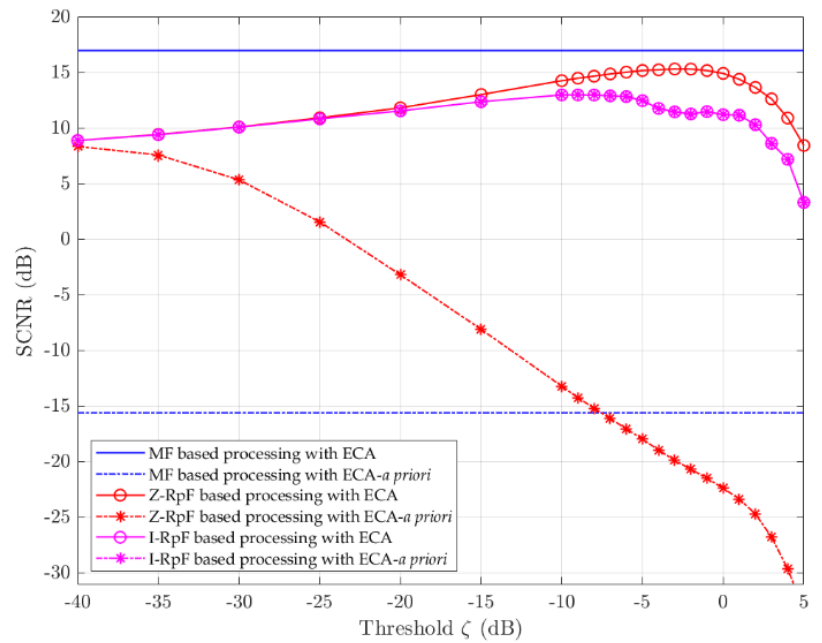

(a)

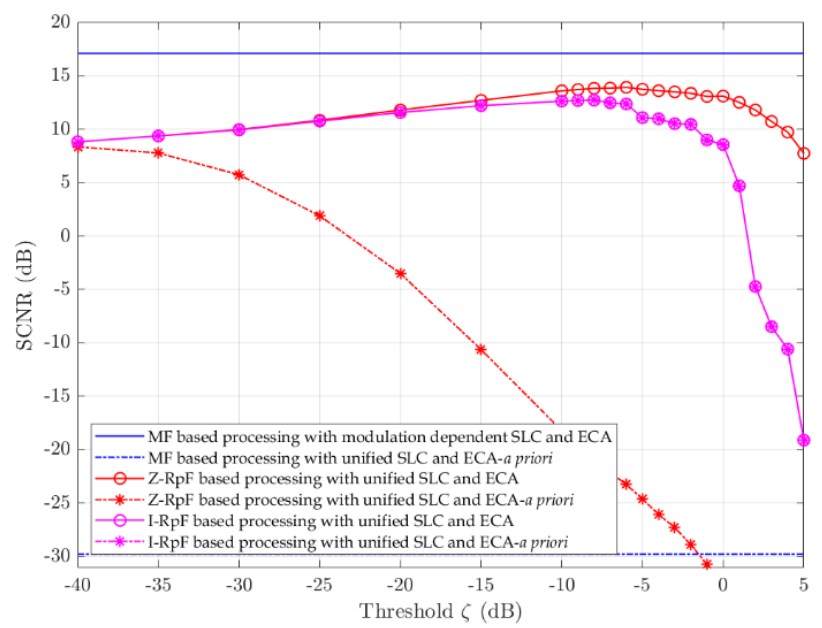

(b)

Fig. 13. SCNR versus threshold for (a) OFDM-only config: (b) $80 \%$ OFDM $-20 \%$ DSSS mixing config.

Therefore, the I-RpF preserves the possibility to operate with the unified processing scheme of Fig. 7 while mitigating the SNR loss yield by the direct application of the RpF.

Consequently, in the following, the I-RpF supervision strategy is considered as the preferred solution and its threshold is set as $\zeta=-10 \mathrm{~dB}$, which yields a SCNR loss of $4 \mathrm{~dB}$ with respect to the MF based modulation dependent processing scheme.

The effectiveness of this selection is proved in Fig. 14 where the resulting unified processing scheme encompassing the ECA-a priori is applied to the same simulated case study reported in Fig. 9. Fig. 14(a) and (b) report the obtained range-velocity maps for the two considered configurations, namely $100 \%$ OFDM packets and $80 \%$ OFDM - 20\% DSSS packets, respectively. As is apparent, in all maps, the clutter cancellation is effective and the background level is around 0 $\mathrm{dB}$. Moreover, with the selected threshold, the target loss is of approx. $4 \mathrm{~dB}$ for all targets, if compared to the results in Fig. 9(a) and (b).

It is worth recalling that the limited loss is obtained with a substantial reduction in the processing chain complexity, especially when dealing with mixed modulation packets, that would otherwise need a modulation recognition stage and ad hoc processing blocks. 


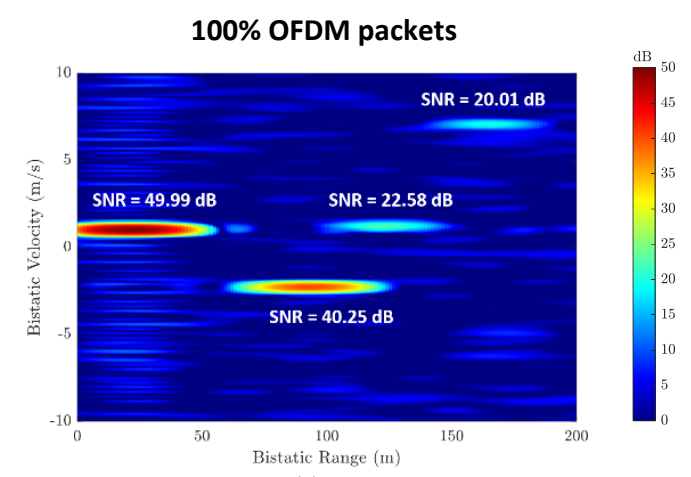

(a)

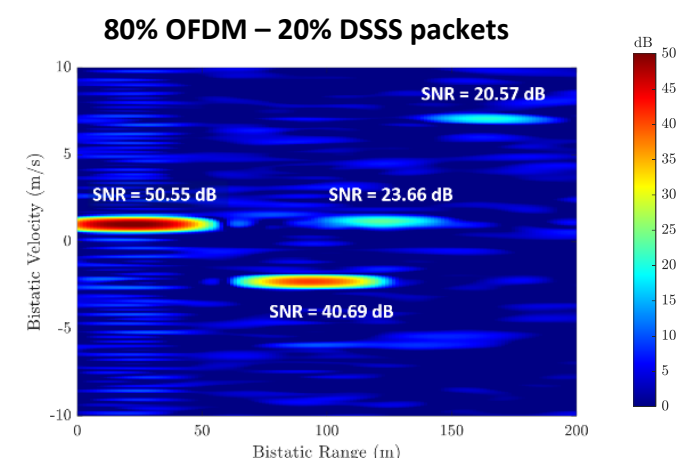

(b)

Fig. 14. Range-velocity map for the simulated scenario with different packet configurations when applying the I-RpF based processing with unified SLC, ECA-a priori and $\zeta=-10 \mathrm{~dB}$

\section{EXPERIMENTAL VALIDATION}

A dedicated acquisition campaign has been carried out in June 2021. The acquisition geometry is reported in Fig. 15(a) where the WiFi AP and the receiver antennas are shown. A commercial wireless AP (TP-Link Archer VR600 AC1600), was used as transmitter of opportunity and connected to a transmitting directive antenna. The AP was configured to transmit packets according to the $802.11 \mathrm{n}$ only standard in channel 13 of the WiFi band $\left(f_{0}=2.472 \mathrm{GHz}, \lambda=\right.$ $0.1214 \mathrm{~m}$ ) with a nominal beacon interval (BI) of $100 \mathrm{~ms}$. Three TP-Link TL-ANT2409A antennas were employed and steered towards the monitored area to collect the surveillance signal. In this work, we consider the data extracted from the right-hand antenna in Fig. 15(a). Two people were present and acted as cooperative human targets walking in the monitored area (Fig. 15(b)). One was moving away from the Rx-Tx pair (Target 1), while the other was moving in the opposite direction (Target 2).

In this work, we show the results for one scan obtained with a CPI of 0.3 seconds, during which 764 packets are collected. During the considered CPI, 759 packets $(99.35 \%)$ are with an OFDM modulation whilst $5(0.65 \%)$ are with a DSSS modulation. In Fig. 16 the range-velocity maps obtained for the considered scan are reported when applying the different processing schemes described and extensively compared in this paper. In all cases, a sliding approach for the clutter cancellation has been considered, resorting to either a conventional ECA-S [37] or its a priori version, with a time interval of 0.05 seconds for the estimation of the cancellation filter coefficients and an update rate corresponding to the packet rate. Note that all maps have been scaled for the output noise level to be around $0 \mathrm{~dB}$.
The results obtained on experimental data confirm what has been already demonstrated with extensive simulated analyses, namely that:

- When the modulation dependent MF based signal processing scheme is applied (see Fig. 16(a)), the highest SNR is obtained for both targets. On the other hand, the reduced range SLC capability is evident, especially for the strongest target (Target 1) and this might yield masking effects on lower RCS targets. This is due to the predominance of OFDM packets.

- When the unified RpF based signal processing scheme of Fig. 2 is applied (see Fig. 16Fig. 14(b)), a target SNR loss is experienced. In this case, the loss is of 10.3 and $11.1 \mathrm{~dB}$ for Target 1 and Target 2 respectively. However, we incidentally notice that this does not represent a significant limitation in the case at hand since the target detection performance is not noiselimited but rather clutter-limited. Moreover, as expected, with the $\mathrm{RpF}$ the range sidelobes are clearly better controlled and overall a lower background level is obtained.

- When the unified I-RpF based signal processing scheme is applied (see Fig. 16(c)) with a properly selected threshold $\zeta=-10 \mathrm{~dB}$, the SNR loss is reduced to its minimum value. Specifically, the final SNR loss with respect to the MF based processing scheme is around 3$4 \mathrm{~dB}$ for both targets. Furthermore, the SLC and the clutter cancellation capability are effectively maintained using the simpler unified processing scheme and employing the a priori version of the ECA-S. This is clearly apparent from the resulting background level which appears to be pretty much controlled. These results match with the theoretical curves in Fig. 12 and Fig. 13.

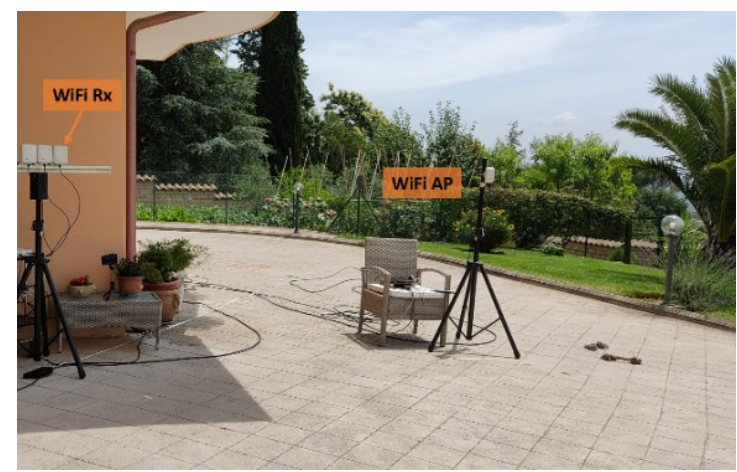

(a)

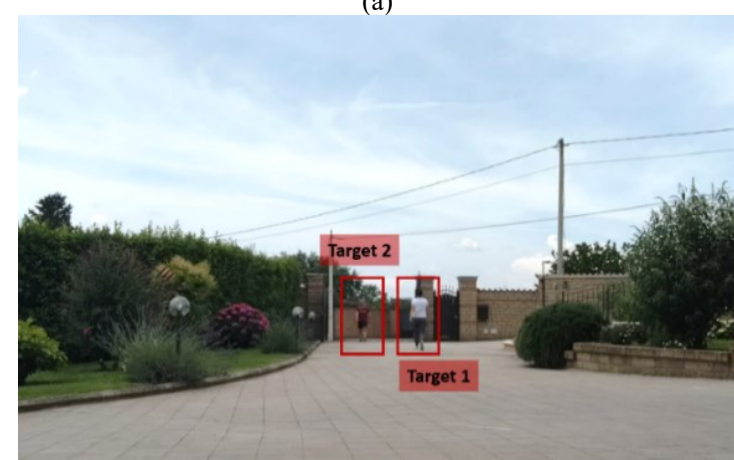

(b)

Fig. 15. Acquisition Campaign: (a) geometry (b) moving targets. 


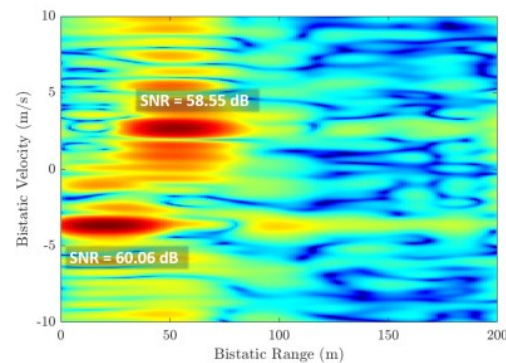

(a)

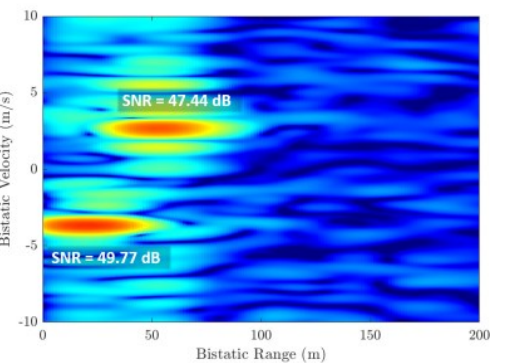

(b)

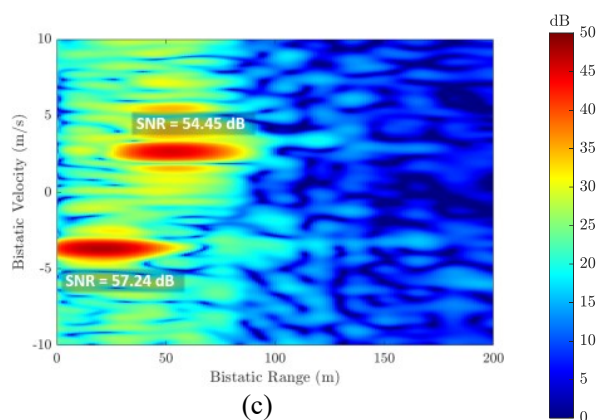

Fig. 16. Range Doppler map for the real dataset with $\mathrm{CPI}=0.3 \mathrm{~s}$ using

(a) MF based processing scheme with modulation dependent SLC and ECA-S

(b) RpF based processing scheme with unified SLC and ECA-S-a priori

(c) I-RpF based processing scheme with unified SLC, ECA-S- a priori and $\zeta=-10 \mathrm{~dB}$.

\section{CONCLUSIONS}

In this work, we devised a novel and simplified signal processing for WiFi-based passive radars. First, we adjusted the conventional signal processing architecture in order to reduce the computational load and simplify the data management. Such simplification is obtained based on the pulsed nature of these transmissions of opportunity by range compressing the data first and then cascading the cancellation block and the Doppler processing. Then, we investigated the possibility of using the $\mathrm{RpF}$ for range compression stage in lieu of the MF and we studied its impact on the overall processing chain. In particular, the use of the $\mathrm{RpF}$ has been demonstrated to allow an effective sidelobes control by resorting to conventional tapering function and to enable an efficient clutter cancellation strategy, referred to as ECA- $a$ priori and regarded as a direct consequence of the equalization of the transmitted signal spectrum yield by the RpF. Finally, appropriate supervision strategies have been proposed to handle the inherent SNR loss obtained with the theoretical RpF. The effectiveness of the proposed approaches has been demonstrated against both simulated and experimental data, showing that the resulting simplified signal processing scheme represents a viable solution in practical applications.

\section{ACKNOWLEDGMENT}

The authors gratefully acknowledge many colleagues at Sapienza and UCL for the helpful comments and stimulating discussions. Among them, a special thanks to Dr. C. Bongioanni for the support in the experimental tests.

\section{REFERENCES}

[1] Special Issue on Passive Radar Systems, IEE Proceedings on Radar, Sonar and Navigation, vol. 152, Issue 3, pp. 106-223, June 2005.

[2] A. Farina, H. Kuschel, "IEEE Aerospace and Electronic Systems Magazine", Special Issue on Passive Radar Part I \& II, vol. 27, pp. 10$11,2012$.

[3] P. Lombardo and F. Colone, "Advanced processing methods for passive bistatic radar," in Melvin, W. L., and Scheer, J. A. (Eds.): 'Principles of Modern Radar: Advanced Radar Techniques', Raleigh, NC: SciTech Publishing, 2012, pp. 739-821.

[4] F. Colone, "Short-range passive radar potentialities" in "Novel Radar Techniques and Applications Volume 1: Real Aperture Array Radar, Imaging Radar, and Passive and Multistatic Radar", Ch. 15, pp. 661718
[5] P. Samczynski, et al., "Passive Radar as a Part of Critical Infrastructure Protection System," 2018 International Conference on Radar, Brisbane, QLD, 2018, pp. 1-5.

[6] D. Cristallini, et al. "Space-Based Passive Radar Enabled `By The New Generation Of Geostationary Satellites", IEEE Aerospace Conference, Big Sky (MT), USA, 6-13 March 2010

[7] A. G. Stove, M. S. Gashinova, S. Hristov and M. Cherniakov, "Passive Maritime Surveillance Using Satellite Communication Signals," in IEEE Transactions on Aerospace and Electronic Systems, vol. 53, no. 6, pp. 2987-2997, Dec. 2017.

[8] F. Filippini, O. Cabrera, C. Bongioanni, F. Colone, P. Lombardo, "DVBS based Passive Radar for Short Range Security Application", 2021 IEEE Radar Conference, Atlanta, USA, 2021.

[9] F. Santi, F. Pieralice and D. Pastina, "Joint Detection and Localization of Vessels at Sea With a GNSS-Based Multistatic Radar," in IEEE Transactions on Geoscience and Remote Sensing, vol. 57, no. 8, pp. 5894-5913, Aug. 2019.

[10] A. A. Salah et al., "Feasibility study of LTE signal as a new illuminators of opportunity for passive radar applications," 2013 IEEE International $R F$ and Microwave Conference (RFM), 2013, pp. 258-262.

[11] A. Evers and J. A. Jackson, "Analysis of an LTE waveform for radar applications," 2014 IEEE Radar Conference, 2014, pp. 0200-0205.

[12] S. Bartoletti, A. Conti and M. Z. Win, "Passive radar via LTE signals of opportunity," 2014 IEEE International Conference on Communications Workshops (ICC), 2014, pp. 181-185.

[13] O. Naparstek and A. Nehorai, "Hybrid opportunistic radar over long term evolution networks," 2015 IEEE Radar Conference (RadarCon), 2015, pp. 1551-1554.

[14] R. S. Thoma et al., "Cooperative Passive Coherent Location: A Promising 5G Service to Support Road Safety," in IEEE Communications Magazine, vol. 57, no. 9, pp. 86-92, September 2019.

[15] P. Falcone, F. Colone, P. Lombardo, "Potentialities and challenges of WiFi-based passive radar", IEEE Aerosp. Electron. Syst. Mag., vol. 27, no. 11, pp. 15-26, Nov. 2012.

[16] https://isac.committees.comsoc.org/? ga=2.120925832.1260253727.16 $23311577-257418631.1620831297$

[17] F. Colone, K. Woodbridge, H. Guo, D. Mason and C. J. Baker, "Ambiguity Function Analysis of Wireless LAN transmissions for passive radar", IEEE Trans. on Aerospace and Electronic Systems, Vol. 47, Issue 1, January 2011, pp. 240-264.

[18] F. Colone, P. Falcone, C. Bongioanni, P. Lombardo, "WiFi-Based Passive Bistatic Radar: Data Processing Schemes and Experimental Results". IEEE Transactions on Aerospace and Electronic Systems. 2012; vol. 48(2), pp. 1061-1079.

[19] K. Chetty, G. E. Smith and K. Woodbridge, "Through-the-Wall Sensing of Personnel Using Passive Bistatic WiFi Radar at Standoff Distances," in IEEE Transactions on Geoscience and Remote Sensing, vol. 50, no. 4, pp. 1218-1226, April 2012.

[20] P. Falcone, F. Colone, A. Macera, and P. Lombardo, "Two-dimensional location of moving targets within local areas using WiFi-based 
multistatic passive radar", IET Radar Sonar Navig., vol. 8, no. 2, pp. 123-131, Feb. 2014.

[21] B. Tan, K. Woodbridge, K. Chetty, "A real-time high resolution passive WiFi Doppler-radar and its applications", 2014 International Radar Conference (Radar), 13-17 Oct. 2014, Lille (France).

[22] D. Pastina, F. Colone, T. Martelli and P. Falcone, "Parasitic Exploitation of Wi-Fi Signals for Indoor Radar Surveillance," in IEEE Transactions on Vehicular Technology, vol. 64, no. 4, pp. 1401-1415, April 2015.

[23] S. Rzewuski, K. Kulpa, P. Samczyński, "Duty factor impact on WIFIRAD radar image quality", 2015 IEEE Radar Conference, 2015, pp. $400-405$.

[24] F. Colone, T. Martelli, C. Bongioanni, D. Pastina, P. Lombardo "WiFibased PCL for monitoring private airfields," IEEE Aerospace and Electronic Systems Magazine, 2017; vol. 32(2), pp. 22-29

[25] H. Sun, L. G. Chia and S. G. Razul, "Through-Wall Human Sensing with WiFi Passive Radar," in IEEE Transactions on Aerospace and Electronic Systems, doi: 10.1109/TAES.2021.3069767.

[26] P. Falcone, F. Colone, P. Lombardo, T. Bucciarelli, "Range Sidelobes Reduction Filters for WiFi-Based Passive Bistatic Radar," EURAD 2009, Rome, Italy, 30 September-2 October 2009.

[27] P. Falcone, F. Colone, C. Bongioanni and P. Lombardo, "Experimental results for OFDM WiFi-based passive bistatic radar," 2010 IEEE Radar Conference, 2010, pp. 516-521.

[28] F. Colone, P. Falcone and P. Lombardo, "Passive Bistatic Radar based on mixed DSSS and OFDM WiFi transmissions," 2011 8th European Radar Conference, 2011, pp. 154-157.

[29] P. Falcone, F. Colone, P. Lombardo, "Doppler frequency sidelobes level control for WiFi-based passive bistatic radar". Proc. 2011 IEEE Radar Conf., Kansas City, MO, USA, May 2011, pp. 435-440.
[30] M. Glende, "PCL-signal-processing for sidelobe reduction in case of periodical illuminator signals", Proc. Int. Radar Symp., pp. 1-4, 2006.

[31] S. Searle, J. Palmer, L. Davis, D. O'Hagan and M. Ummenhofer, "Evaluation of the ambiguity function for passive radar with OFDM transmissions", Proc. IEEE Radar Conf., pp. 1040-1045, 2014.

[32] J. Palmer, H. Harms, S. Searle and L. Davis, "DVB-T passive radar signal processing", IEEE Trans. Signal Process., vol. 61, no. 8, pp. 2116-2126, Apr. 15, 2013.

[33] G. Gassier et al., "A unifying approach for disturbance cancellation and target detection in passive radar using OFDM", IEEE Trans. Signal Process., vol. 64, no. 22, pp. 5959-5971, Nov. 2016.

[34] G. Chabriel and J. Barrere, "Adaptive target detection techniques for OFDM-based passive radar exploiting spatial diversity", IEEE Trans. Signal Process., vol. 65, no. 22, pp. 5873-5884, Nov. 2017.

[35] P. Wojaczek, F. Colone, D. Cristallini and P. Lombardo, "ReciprocalFilter-based STAP for passive radar on moving platforms," IEEE Transactions on Aerospace and Electronic Systems, vol. 55, no. 2, pp. 967-988, April 2019

[36] Wireless LAN Medium Access Control (MAC) and physical Layer (PHY) Specifications, IEEE Std. 802.11, 2016.

[37] F. Colone, C. Palmarini, T. Martelli, E. Tilli, "Sliding extensive cancellation algorithm for disturbance removal in passive radar", in IEEE Transactions on Aerospace and Electronic Systems, vol. 52, no. 3, pp. 1309-1326, June 2016. 